\title{
VOLUME CONDUCTION AND EEG MEASUREMENTS WITHIN THE BRAIN: A QUANTITATIVE APPROACH TO THE INFLUENCE OF ELECTRICAL SPREAD ON THE LINEAR RELATIONSHIP OF ACTIVITY MEASURED AT DIFFERENT LOCATIONS
}

\author{
J. HOLSHEIMER * and B.W.A. FEENSTRA **
}

Bio-information Group, Department of Electrical Engineering, Twente University of Technology, Enschede (The Netherlands)

(Accepted for publication: November 9, 1976)

When measuring brain field potentials within the EEG frequency range $(0-50 \mathrm{c} / \mathrm{sec})$ it is in general difficult to interpret the recorded signal in terms of the site of the underlying neuronal processes (Malliani et al. 1965). One often tries to overcome this type of difficulty by measuring the EEG activity simultaneously at different sites within a brain structure and by determining the relationship between the different signals (Abraham et al. 1973; Winson 1974, 1976; Bland et al. 1975). This relationship can be mediated by two different processes. One is caused by direct or indirect neuronal connections between the populations of neurones from which the records are made, resulting in linearly or non-linearly related signals (Lopes da Silva et al. 1973; Ganes and Andersen 1975).

The process always involved is volume conduction: the local ionic current through an active nervous membrane causes an electrical field in the surrounding tissue. Volume conduction, or electrical spread, is a passive resistive process: the amplitude of the extracellular signal decreases as the distance from the

* Reprint requests to J. Holsheimer, Dept of Electrical Engineering, Twente University of Technology, P.O. Box 217, Enschede, The Netherlands.

** Present address: Dept. of Medical and Physiological Physics, Physics Laboratory, State University Utrecht, The Netherlands. active membrane increases. As a result of volume conduction one can measure the electrical activity of a neurone at some distance from its membrane. This process makes it possible to measure the electrical activity of neurones situated at some distance from the electrode and hence to measure the summed activity of a population of neurones around the electrode. But by this process it is also possible that active neurones contribute to the signal of more than one electrode, thus contributing to the linear dependence of these electrode signals. The influence of volume conduction on the linear relationship between the recorded signals may be considerable, especially when measuring with electrodes having a small tip separation.

Thus the influence of volume conduction may be a disturbing factor in determining the relation between the populations of neurones surrounding recording electrodes as a result of neuronal interaction. In order to quantify the linear relation of neuronal activity being generated by neurone populations in different parts of the brain it is desirable to eliminate or account for the possible linear relation resulting from volume conduction.

In this article we describe a method to quantify the linear relationship between two recorded signals due to electrical spread in a situation with independent neuronal sources. 


\section{Materials and methods}

Five female Wistar rats $(210-250 \mathrm{~g})$ were used under urethane anaesthesia (1.1-1.4 $\mathrm{g} / \mathrm{kg}$ ). Urethane was chosen because this anaesthetic, in the concentration applied, appeared to favour spontaneous theta activity in the hippocampus. Measurements were carried out in different parts of the hippocampus, including the fascia dentata, against a reference electrode - the stereotactic apparatus. During the experiments the body temperature of the rat was kept constant at about $38^{\circ} \mathrm{C}$, using an electronically controlled heating coil.

The recording electrodes were made of glass-coated $50 \mu$ platinum wire. The whole wire except the tip surface was insulated. The real surface of the semi-microelectrodes was $0.0019 \mathrm{~mm}^{2}$, and the impedance $2-10 \mathrm{M} \Omega$ at $10 \mathrm{c} / \mathrm{sec}$. FET amplifiers developed in our laboratory, having an input impedance of $10^{4}$ $\mathrm{M} \Omega$ at $10 \mathrm{c} / \mathrm{sec}$ were used (Bergveld and Holsheimer 1971). The electrode holder was attached to a rat stereotactic apparatus (Minderhoud 1968) and consisted of two microdrives with their axes at an angle of $17.5^{\circ}$ in such a way that the point of intersection of the electrodes corresponded with the dorsoventral zero plane of the stereotactic apparatus.

During the experiments one electrode was fixed at the point of intersection and the other one was moved, resulting in a series of EEG records on analog tape at different sites and electrode tip separations.

Frequency analysis of $8 \mathrm{sec}$ EEG epochs was carried out on a PDP-11 computer, using an FFT algorithm. As an estimate of the linear relationship between the stochastic signals in each record the squared coherence function was computed (Jenkins and Watts 1968). Power spectra and coherence function were computed in the frequency range of $0-64 \mathrm{c} / \mathrm{sec}$ and plotted from $1.5-30 \mathrm{c} / \mathrm{sec}$ with a frequency resolution of $1 / 8 \mathrm{c} / \mathrm{sec}$. Frequency averaging was employed, using a Papoulis window consisting of 13 frequency points (Papoulis 1973). Apart from this the mean value of coherence in the frequency band of $12-30 \mathrm{c} / \mathrm{sec}\left(\mathrm{coh}_{\mathrm{m}}\right)$ was calculated. The advantage of taking this mean value above the coherence at one frequency in this range is that $\operatorname{coh}_{m}$ is a much more reliable estimate, especially at low values of coherence which have a large variance. Since the frequency band of $12-30 \mathrm{c} / \mathrm{sec}$ comprises 14 independent estimates of coherence, this averaging gave rise to a reduction in variance of $1 / 14$. The estimates of coherence were biascorrected, corresponding to the linear approximation of Benignus (1969).

\section{Results}

Since we were interested in the linear relationship between rhythmic activity at different sites of a brain structure, i.e. theta activity in the hippocampal complex, only spectra and coherence of epochs showing theta activity were computed. The spectra had a peak at $4.0-5.0 \mathrm{c} / \mathrm{sec}$ and sometimes also at the second harmonic $(8-10 \mathrm{c} / \mathrm{sec})$. This decrease in theta frequency under urethane anaesthesia in comparison with the normal of $7-9 \mathrm{c} / \mathrm{sec}$ in rat (Whishaw and Vanderwolf 1973) was similar to the effect of urethane on eserine-induced theta activity found by Stumpf et al. (1962).

Coherence functions had different shapes at different electrode separations. In general coherence in the theta frequency band (and its second harmonic) had a high value at most interelectrode distances, whereas coherence outside this frequency range had a value which decreased with increasing electrode tip separation.

It is well known that during theta activity the membrane potential fluctuations of most pyramidal cells in the hippocampus show rhythmic activity and that these cells are considered to be the main sources of the hippocampal EEG (Fujita and Sato 1964). Since large populations of interconnected pyramidal cells are involved, which give rise to this synchronized rhythmic activity, we may con- 
clude that the process of neuronal interaction will be limited to the theta frequency range (and its second harmonic). This process is characterized by the peaks in the power spectrum. Assuming that, except for these peaks, there is little correlation between the activity of different pyramidal cells, we may consider the linear relationship between the recorded signals outside the theta frequency range (and its second harmonic) as a result of electrical spread only. Therefore we took the main value of coherence in the frequency band of $12-30 \mathrm{c} / \mathrm{sec}, \mathrm{coh}_{\mathrm{m}}$, as an estimate of linear relationship of the recorded signals due to the passive process of volume conduction.

The influence of electrode separation on $\mathrm{coh}_{m}$ is shown in Fig. 1 for six experiments. The value of $\operatorname{coh}_{m}$ decreases as a function of electrode tip separation, resulting in a negligible linear relationship $\left(\mathrm{coh}_{\mathrm{m}}<0.10\right)$ at a distance varying from $0.8-1.4 \mathrm{~mm}$. The differences between the curves must be attributed to different electrical properties (inhomogenities, anisotropy) of the tissue between the two electrodes in the various experiments, since the electrode trajectories were different in all experiments. For this reason it does not make sense to calculate a mean of all curves of $\mathrm{coh}_{\mathrm{m}}$ as a standard estimate for volume conduction in hippocampal tissue, and even less in grey matter in general. Each electrode traject will have its specific curve as a result of specific tissue properties, which lead to different electrical spread characteristics.

The conclusion which may be drawn from Fig. 1 is that the largest distance at which activity in the frequency band of $12-30 \mathrm{c} / \mathrm{sec}$ of hippocampal neurones contributes to the recorded signal will be half the electrode separation at $\operatorname{coh}_{\mathrm{m}}=0.1$, being $0.4-0.7 \mathrm{~mm}$. The coherence level of 0.1 reflects a value of -25 $\mathrm{dB}$ for the ratio : power of common signal of the two records/total power of the recorded signal. Besides coherence as a result of volume conduction $\left(\mathrm{coh}_{\mathrm{m}}\right)$ the coherence resulting from volume conduction plus neuronal interaction during theta activity (coherence at the theta peak frequency, $\operatorname{coh}_{\theta}$ ) from one experi-



Fig. 1. Curves of $\operatorname{coh}_{\mathrm{m}}(12-32 \mathrm{c} / \mathrm{sec})$ as a function of the electrode interval in six experiments and $\operatorname{coh}_{\theta}$ from one experiment (dashed line).

ment is shown in Fig. 1 by a dashed line. It can be seen that at all electrode separations $\operatorname{coh}_{\theta}$ exceeds the value of $\operatorname{coh}_{\mathrm{m}}$. Thus it can be concluded that at all interelectrode distances in Fig. 1 there is a linear relationship between theta activity of a population of neurones, which in turn has its implications for the volume conduction properties of this type of activity, as will be discussed later.

Model of volume conduction producing linear relationship of recorded signals

We consider the volumes of tissue around the electrode tips $\mathrm{X}$ and $\mathrm{Y}$ within which the neurones contribute to the recorded signals as spheres with radius $\mathrm{r}$ and equal volumes $\mathrm{A}$ and $B$. The overlap of these spheres will depend on the distance $d$ between the centres of the spheres. The volume of overlap $S$ of these spheres is given by $S=2 / 3 \pi\left(r-\frac{1}{2} d\right)^{2}\left(2 r+\frac{1}{2} d\right)$ for $\mathrm{d} \leqslant 2 \mathrm{r}$. The model is depicted in Fig. $2 \mathrm{~A}$. As a first approximation we assume that: the cell density is uniform and the same within both spheres; the mean contributions to the electrode signal of all neurones within the spheres are equal; there is no linear relationship between the stochastic electrical activities of the neurones.

Then the powers of the electrode signals 

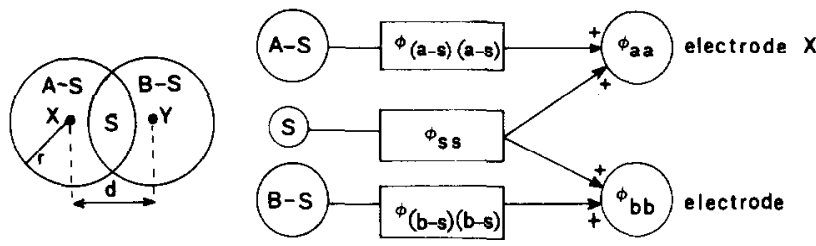

A

B

Fig. 2. Model of volume conduction. A. The spheres $A$ and $B$ with centres being the electrode tips $X$ and $Y$ and radius $r$ will overlap by a volume $S$ when the interval $\mathrm{d}$ between $\mathrm{X}$ and $\mathrm{Y}$ is smaller than $2 \mathrm{r}: \mathrm{S}=$ $2 / 3 \pi(r-0.5 d)^{2}(2 r+0.5 d)$. B. Schematic representation of the signal sources $(A-S),(B-S)$ and $S$, producing power $\phi_{(\mathrm{a}-\mathrm{s})(\mathrm{a}-\mathrm{s})}, \phi_{(\mathrm{b}-\mathrm{s})(\mathrm{b}-\mathrm{s})}$ and $\phi_{\mathrm{ss}}$ respectively. Summation of the independent signals $(A-S)$ and $S$ gives the electrode signal of $X$, summation of $(B-S)$ and $S$ gives the electrode signal of $Y$.

$\phi_{\mathrm{aa}}$ and $\phi_{\mathrm{bb}}$ will be equal and there will be no linear relationship between the signals when there is no overlap of the spheres $(d \geqslant 2 r)$. When $d<2 r$ there will be a common volume $\mathrm{S}$ and common power $\phi_{\mathrm{ss}}$. The ratio $\phi_{\mathrm{ss}} / \phi_{\mathrm{aa}}$ $\left(=\phi_{\mathrm{ss}} / \phi_{\mathrm{bb}}\right)$ will be the same as the ratio of the volumes $\mathrm{S} / \mathrm{A}(=\mathrm{S} / \mathrm{B})$, under the conditions mentioned above. The common power $\phi_{\mathrm{ss}}$ will give rise to a linear relationship between the signals at the electrodes $\mathrm{X}$ and $\mathrm{Y}$, as shown schematically in Fig. 2B. The squared coherence function can be used to estimate the

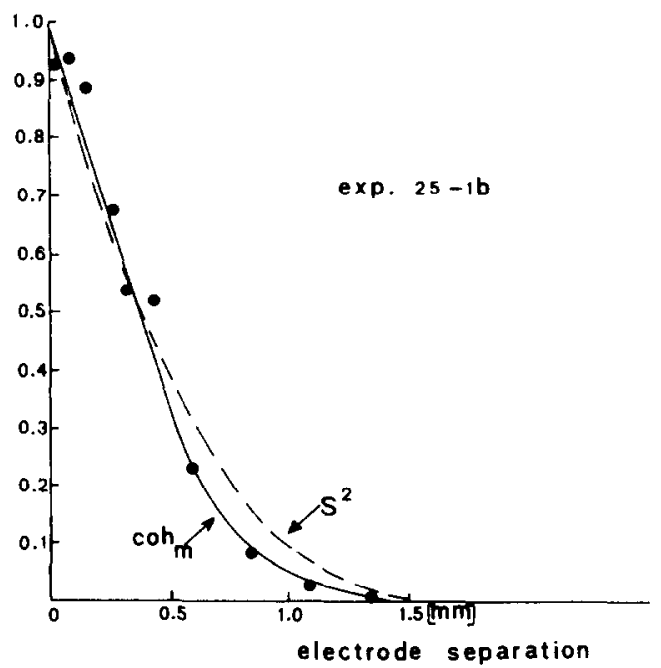

Fig. 3. Curve of $\operatorname{coh}_{\mathrm{m}}$ and the best fitting curve of $\mathrm{S}^{2}$ $(\mathrm{r}=1.0 \mathrm{~mm})$. relative influence of this common source as follows:

$\operatorname{coh}_{\mathrm{XY}}=\frac{\phi_{\mathrm{ss}} \cdot \phi_{\mathrm{ss}}}{\phi_{\mathrm{aa}} \cdot \phi_{\mathrm{bb}}}$

Assuming that $\mathrm{r}$ and $\phi_{\mathrm{aa}}\left(=\phi_{\mathrm{bb}}\right)$ are constant for different electrode positions, then $\operatorname{coh}_{X Y}$ is proportional to $\phi_{\mathrm{ss}}^{2}$ and hence proportional to $\mathrm{S}^{2}$. Since the maximum value of the coherence is 1.0 we also give $S^{2}$ a maximum value of 1.0. This maximum corresponds with the squared volume $\mathrm{A}^{2}\left(=\mathrm{B}^{2}\right)$.

In order to see whether the model gives a good description of the linear relationship between the signals $X$ and $Y$ as a result of volume conduction, we have to compare $\mathrm{coh}_{\mathrm{m}}$ and $\mathrm{S}^{2}$ as a function of the electrode separation $d$ in the experiments. The only parameter is the value of the radius $r$ of the spheres. For an optimal fit of $\mathbf{S}^{2}$ with the curves of $\mathrm{coh}_{\mathrm{m}}$, in Fig. 1 the value of $r$ lies between $0.9-1.6$ mm.

Results obtained from one experiment and by means of the model are shown in Fig. 3 . The small discrepancy between the two curves can be accounted for by the assumption in the model that the mean contribution to the electrode signal of all neurones within the spheres should be equal. This assumption was made in order to get a simple quantitative model. However, this is an over-simplification because, according to the theory of volume conduction, the contribution of the different neuronal sources to the recorded signal decreases with increasing distance of the neurone from the electrode. It is useless trying to get a perfect fit of experimental and theoretical curves since other unknown characteristics of the tissue such as electrical (in)homogenity and (an)isotropy affect the volume conduction properties.

\section{Discussion}

In comparison with the frequency range $12-30 \mathrm{c} / \mathrm{sec}$, there is much more power in the frequency range $4-6 \mathrm{c} / \mathrm{sec}$ during theta activ- 
ity and therefore the influence of neurones on the extracellular field will spread over a much larger distance in the latter than in the former frequency range. Coherence functions (in which the relation between the model of Fig. 2 and experiments is expressed), however, do not measure absolute power but the ratio of powers of common and linearly independent sources. So if hippocampal sources generating $4-6 \mathrm{c} / \mathrm{sec}$ and $12-30 \mathrm{c} / \mathrm{sec}$ should be identical, the decay of coherence as a function of electrode separation should be the same for the two frequency ranges. But in the case of theta activity the hippocampal source has a more complex form since theta rhythm is due to synchronous activity of a large neuronal population forming a dipole layer within a warped surface. The difference between the decay of coherence as a function of electrode separation for the two frequency ranges can be accounted for by the difference in size of the respective neuronal sources. In the case of the $12-30 \mathrm{c} / \mathrm{sec}$ range each neurone is a small independent source, as assumed in the simplified model of Fig. 2A, but in the 4-6 $\mathrm{c} / \mathrm{sec}$ range the source is a relatively large dipole layer. So our model will not hold for theta activity. Since the amplitude of the field potential of a dipole reduces with the inverse of the squared distance from source, whereas the field potential of a dipole layer reduces with the inverse of the distance, the power common to the two electrodes, and thus the coherence at $4-6 \mathrm{c} / \mathrm{sec}$, will decay in a much less steeply than at $12-30 \mathrm{c} / \mathrm{sec}$.

For an exact model of the decay of coherence in the $4-6 \mathrm{c} / \mathrm{sec}$ range as a function of electrode separation we should have to know the distribution and orientation of the neurones and the form and intensity of their respective extracellular fields, as investigated by Horowitz and Freeman (1966) for the prepyriform cortex and by van Rotterdam et al. (1974) for the dentate area. But these considerations do not detract from the conclusion from Fig. 1 that the significant linear relationship between the recorded signals at theta frequency is a result of neuronal interac- tion within a population of pyramidal cells.

As synchronized activity of neurones in a cell layer may give rise to a large extracellular field, the activity of such a neurone population may contribute considerably to a signal recorded at a distant place. If there is little neuronal activity in the direct surroundings of the electrode the main contribution to the recorded signal may originate from such a remote source. This problem has already been discussed in the literature (Malliani et al. 1965; Abraham et al. 1973). When recording such a strong remote source with two electrodes against a reference this may result in a large in-phase component in the simultaneous records. In order to distinguish this situation from one with two phase-locked sources in the surroundings of both electrodes, the phase and amplitude relations between the signals in the frequency range of interest can provide more information since volume conduction is a process giving rise to constant phase and continuous decrease of amplitude as a function of distance from the neuronal source (Winson 1974, 1976; Bland et al. 1975; Feenstra and Holsheimer, in preparation).

When recording field potentials in a brain structure with two electrodes against a reference electrode at some inactive site, the possible contribution of electrical spread to coherence between the simultaneously recorded sig. nals can be determined in a situation in which there is no linear relationship between the neurones contributing to the recorded signals. When in some frequency band (for instance at a peak in the power spectrum) coherence has a higher value, this will be the result of neuronal interaction giving rise to synchronisation of cell activities. The volume conduction properties of such a population of synchronized electrical sources will be different from those of independent sources and will also result in an increased coherence. Thus the effects of synchronization of a neuronal population, and the consequent changes in volume conduction properties on the coherence of simultaneously recorded field potentials, cannot be distinguished experimentally. 


\section{Summary}

When recording referentially brain field potentials with several electrodes at relatively small tip separations, a linear relationship between the simultaneously recorded signals may arise solely as a result of volume conduction (electrical spread). A method is described to quantify the linear relationship due to electrical spread in a situation with independent neuronal sources.

In rat under urethane anaesthesia, records were made during theta activity in the hippocampus with two electrodes against a reference with electrode tip separations between 0-3 mm. Frequency analysis of EEG epochs and computation of coherence were carried out.

As an estimate of linear relationship between the recorded signals due to electrical spread the mean value of coherence $\left(\mathrm{coh}_{\mathrm{m}}\right)$ of a frequency band outside the range containing most power of theta rhythm was calculated.

The results show a fairly constant decay of $\mathrm{coh}_{\mathrm{m}}$ at increasing electrode separation, reaching a value of 0.1 at a distance varying between $0.8-1.4 \mathrm{~mm}$. This means that neurones at a distance of $0.4-0.7 \mathrm{~mm}$ from a recording electrode make a contribution of $-25 \mathrm{~dB}$ to a recorded signal of $0 \mathrm{~dB}$.

The results of a simple model of volume conduction producing linear relationship between two recorded signals are in good agreement with the experimental results.

The influence of linear relationship of the activity of neurones on volume conduction properties and on coherence is discussed.

\section{Résume}

Conduction en volume et évaluations de l'EEG dans le cerveau: analyse quantitative de l'influence de la dispersion électrique sur la relation linéaire entre activités recueillies en divers points.

Lorsqu'on enregistre les champs électriques cérébraux par plusieurs électrodes relative- ment proches, par rapport à une référence, une relation linéaire apparaît entre les signaux recueillis simultanément, qui résulte d'une conduction dans le volume (dispersion électrique passive). On décrit une méthode qui permet de quantifier cette relation linéaire, dans le cas de sources neuronales indépendantes.

Des enregistrements du thêta hippocampique ont été effectués chez le rat sous anesthésie à l'uréthane, par deux électrodes dont la distance entre points variait entre 0 et $3 \mathrm{~mm}$, ceci par rapport à une référence. On a procédé à une analyse fréquentielle de segments de tracé, ainsi qu'à une évaluation de cohérence.

Pour évaluer la relation linéare entre signaux qui est due à la conduction en volume, on a calculé la valeur moyenne de la cohérence $\left(\operatorname{coh}_{m}\right)$ dans une bande de fréquence ne contenant pas la puissance maximale du thêta.

Il apparaît alors que $\operatorname{coh}_{\mathrm{m}}$ décroît régulièrement lorsque l'écart entre les électrodes est augmenté, pour atteindre 0,1 pour une distance entre 0,8 et $1,4 \mathrm{~mm}$. Ce qui signifie que des neurones à une distance de 0,4 à $0,7 \mathrm{~mm}$ de l'électrode de dérivation contribuent pour $-25 \mathrm{~dB}$ à un signal recueilli de $0 \mathrm{~dB}$.

Les déductions faites à partir d'un modèle simple de volume conducteur, avec relation linéaire entre deux signaux recueillis, sont en bon accord avec les données expérimentales.

L'influence d'une relation linéaire entre activité neuronales sur les propriétés de volume conducteur et sur la cohérence est discutée.

We are grateful to Dr. F.H. Lopes da Silva and Mr. A. van Rotterdam for suggestions and discussions, to Dr. A. Crowe for correcting the manuscript, to $\mathrm{Mr}$. $\mathrm{H}$. Wijnmaalen for preparing the recording electrodes, to Miss T. Steijlen for typing the manuscript.

\section{References}

Abraham, F., Bryant, H., Mettler, M., Bergerson, B., Moore, F., Maderdrut, J., Gardinier, M., Walter, D. and Jennrich, R. Spectrum and discriminant analyses reveal remote rather than local sources for 
hypothalamic EEG: could waves affect unit activity? Brain Res. 1973, 49:349-366.

Benignus, V.A. Estimation of the coherence spectrum and its confidence interval using the fast fourier transform. IEEE trans. on audio and electroacoustics, 1969, AU-17:145-150.

Bergveld, P. and Holsheimer, J. An unipolar recording system using a microprobe with built-in FET's and remote amplifier. Report Bio-information Group, Department of Electrical Engineering, Twente University of Technology, Enschede, The Netherlands, $1971,9 \mathrm{p}$.

Bland, B.H., Andersen, P. and Ganes, T. Two generators of hippocampal theta activity in rabbits. Brain Res., 1975, 94: 199-218.

Fujita, Y. and Sato, T. Intracellular records from hippocampal pyramidal cells in rabbit during theta rhythm activity. J. Neurophysiol., 1964, 27: $1011-1025$.

Ganes, T. and Andersen, P. Barbiturate spindle activity in functionally corresponding thalamic and cortical somato-sensory areas in the cat. Brain Res., 1975, 98: 457--472.

Horowitz, J.M. and Freeman, W.J. Evoked potentials arising from neural population elements excited at different times on a warped surface. Bull. Math. Biophysics, 1966, 28: 519-536.

Jenkins, G.M. and Watts, D.G. Spectral analysis and its applications. Holden-Day, San Fransisco, 1968, $525 \mathrm{p}$.

Lopes da Silva, F.H., van Lierop, T.H.M.T., Schrijer, C.F. and Storm van Leeuwen, W. Organization of thalamic and cortical alpha rhythms: spectra and coherences. Electroenceph. clin. Neurophysiol, $1973,35: 627-639$.
Malliani, A., Rudomin, P. and Zanchetti, A. Contribution of local activity and electric spread to somatically evoked potentials in different areas of the hypothalamus. Arch. ital. Biol., 1965, 103: 119135.

Minderhoud, J.M. An "all-round" apparatus for stereotactic operations on small test animals (albino rat). Brain Res., 1968, 9: 380-384.

Papoulis, A. Minimum-bias windows for high-resolution spectral estimates. IEEE trans. on inf. theory, 1973, IT-19:9-12.

Rotterdam, van A., Habets, A. and Wouters, W. Field potentials in the dentate area of the cat - experimental findings and biophysical models. Progress Report Inst. Med. Phys., Utrecht, The Netherlands, 1974 , pr. $4: 104-113$.

Stumpf, Ch., Petsche, H and Gogolak, G. The significance of the rabbit's septum as a relay station between the midbrain and the hippocampus. II. The differential influence of drugs upon both the septal cell firing pattern and the hippocampus theta activity. Electroenceph. clin. Neurophysiol. $1962,14: 212-219$.

Whishaw, I.Q. and Vanderwolf, C.H. Hippocampal EEG and behavior: changes in amplitude and frequency of RSA (theta rhythm) associated with spontaneous and learned movement patterns in rats and cats. Behav. Biol., 1973, 8: 461-484.

Winson, J. Patterns of hippocampal theta rhythm in the freely moving rat. Electroenceph. clin. Neurophysiol., 1974, 36: 291-301.

Winson, J. Hippocampal theta rhythm. I. Depth profiles in the curarized rat. Brain Res., 1976, 103: $57-70$. 